\title{
Política ambiental y su impacto en la innovación tecnológica y organizativa: el reciclaje de vehículos automotores
}

\author{
Ma. de Lourdes Álvarez Medina*
}

\section{Resumen}

Nuevos campos de estudio, como la ecología industrial, han influido en la preocupación de la sociedad por la conservación del medio ambiente y en el establecimiento de políticas que eviten el daño ambiental. Este trabajo analiza cómo estos conceptos se ven reflejados en las politicas ambientales de los países y en el comportamiento estratégico de las empresas. Se revisa la Directiva 2000/54/EC de la Unión Europea que regula el reciclaje de vehículos automotores y la influencia que ha tenido sobre trayectorias de innovación en el diseño de productos y procesos, así como en las nuevas relaciones de cooperación y organización de la industria de reciclaje. Es importante estudiar la problemática de aplicación y los efectos de esta nueva política ambiental porque en México también existen graves problemas de contaminación por vehículos automotores que no se reciclan adecuadamente. Además, en nuestro país, el problema se incrementará al entrar en vigor los acuerdos sobre importación de vehículos usados establecidos en el Tratado de Libre Comercio de América del Norte. El problema de contaminación puede verse como una oportunidad para incentivar el crecimiento de varias industrias, beneficiarse de inversiones y crear empleo. El reto está en que la sociedad civil, el gobierno y las empresas cooperen para crear la regulación y acuerdos necesarios para aprovechar esta oportunidad y cuidar la calidad del medio ambiente.

Palabras clave: ecología industrial, reciclaje automotriz, México, Unión Europea.

\section{Introducción}

— $\mathrm{n}$ las últimas décadas, la preocupación por la conservación del medio ambienEte ha llevado a la sociedad civil, a los gobiernos de los países y a las empresas a establecer mecanismos de regulación directa e indirecta que eviten el daño ambiental.

\footnotetext{
*Investigadora de la División de Investigación de la Facultad de Contaduría y Administración, UNAM. Correo electrónico: malvarez@server.contad.unam.mx
} 
Han emergido conceptos como la responsabilidad del productor, el principio de equidad que señala que "el que contamina paga", el análisis del ciclo de vida del producto y los productos con múltiples ciclos de vida. Tales nociones han influido en la regulación y en las trayectorias de innovación tecnológica y organizativa de las industrias. Nuevos campos de estudio como la ecología industrial buscan comprender el funcionamiento de las mismas.

La contaminación por vehículos automotores ha sido una de las preocupaciones fundamentales de la regulación ambiental desde hace muchos años. Ésta se ha enfocado principalmente en la disminución de emisiones contaminantes al aire, para lo que ha sido necesario la reducción del peso del automóvil y del uso de combustible. Sin embargo, al llegar al final de su vida útil los restos del vehículo son altamente contaminantes si no se reciclan adecuadamente. En los países de la Unión Europea esta problemática se empezó a discutir a principios de la década de los noventa y en septiembre del 2000 el Parlamento de la Unión Europea ${ }^{1}$ aprobó la Directiva ${ }^{2}$ 2000/54/EC que regula el tratamiento de los vehículos automotores que llegan al final de su vida útil (ELV, por sus siglas en inglés: End of Life Vehicles).

La Directiva contiene instrumentos de regulación directa e indirecta que se aplican de forma diferente en cada país debido a que sus leyes, acuerdos y capacidades son heterogéneos, lo que le da un alto grado de complejidad. Las innovaciones tecnológicas que se incentivan se relacionan con el diseño para desensamblar y el diseño para reciclar, mientras que las organizativas consisten en actividades de coordinación y cooperación entre los diferentes actores para hacer crecer y fortalecer a una industria de reciclaje incipiente.

Estudiar los resultados de esta Directiva ambiental es importante porque en México también existen problemas muy graves de contaminación por ELV. Además, el problema de la contaminación por vehículos automotores se acrecentará con la entrada en vigor de los acuerdos del Tratado de Libre Comercio para América del

\footnotetext{
${ }^{1}$ El Parlamento Europeo representa a los pueblos de los estados reunidos en la Comunidad Europea a través de 626 diputados y ejerce tres competencias: legislativas, presupuestarias y de control. El parlamento adopta la legislación comunitaria junto con el Consejo Europeo, compuesto por los jefes de Estado y el Presidente de la Comisión.

${ }^{2}$ Una Directiva, ley para la Unión Europea, obliga a los estados miembros a cumplir con determinados resultados, pero les permite elegir los medios. Esto quiere decir que cada Estado puede crear diferentes leyes que apoyen las directivas siempre y cuando se cumpla con los resultados determinados en ésta.
} 
Norte (TLCAN), que establece que a partir de 2009 se podrán importar de la zona de TLCAN, vehículos con diez años de antigüedad o más, y la permisividad de antigüedad disminuirá progresivamente hasta que en el 2019 se pueda importar todo tipo de vehículos. ${ }^{3}$ Esto representará un incremento exponencial del parque vehicular en México y una serie de problemas y oportunidades que deben preverse. Para diseñar los medios para solucionar dichos problemas, puede ser útil analizar el proceso de implementación y los resultados que ha tenido la Directiva en la Unión Europea y extraer algunas lecciones para nuestro país.

Aquí se analiza cómo la política ambiental, que regula el reciclaje de los vehículos que llegan al final de su vida útil, estimula la innovación en productos, procesos y organización de la estructura de la industria automotriz en la Unión Europea. La información será útil para México debido a que enfrenta graves problemas con los ELV. El artículo se ha organizado en cuatro partes: en primer lugar se retoman conceptos teóricos sobre ecología industrial que se ven reflejados en la legislación y en las actividades estratégicas de las empresas; a continuación se describen los puntos más importantes presentados en la Directiva 2000/54/EC y las acciones dirigidas a cambiar el diseño y producción de automóviles, además se analizan algunos cambios en la organización de la industria de reciclaje, especificando las innovaciones y mercados que se promueven; en tercer lugar se presentan algunos problemas y situaciones del reciclaje de vehículos automotores en México; finalmente, se presentan algunas conclusiones y recomendaciones.

En este trabajo se utilizaron las investigaciones realizadas por el Joint Research Center y el Institute for Prospective of Technological Studies, hechas por encargo de la Comisión Europea sobre ELV; estadísticas de laAssociation des Constructeurs Europeen d' automobiles; el Diario Oficial de la Unión Europea; libros, revistas especializadas y artículos que se encuentran en Internet; así como entrevistas a directivos de empresas y funcionarios públicos en México.

\section{Un nuevo paradigma ambiental}

En las últimas décadas, la variable ambiental se integra como un elemento estratégico en la dirección de las empresas. Esto incluye una filosofía empresarial de

\footnotetext{
${ }^{3}$ Secretaría de Comercio y Fomento Industrial, "Decreto de promulgación del Tratado de Libre Comercio de América del Norte", México, Diario Oficial de la Federación, 20 de diciembre de 1993, p. 45.
} 
desarrollo sustentable, sistemas tecnológicos limpios y énfasis en reutilización y reciclaje. En general, se busca la implantación de estrategias que eviten los problemas. Esta visión ha retomado conceptos de la ecología industrial, que concibe la calidad ambiental en términos de la interacción del ambiente natural y del económico con un enfoque especial en flujo de materiales y uso de energía. Su evaluación se lleva a cabo a través de las actividades de producción y consumo porque el impacto ambiental está en función de cómo se produce, distribuye, emplea y se dispone de los bienes. ${ }^{4}$

La visión ambiental se puede traducir a ventajas competitivas al disminuir el consumo de materiales y energía, reusar o reciclar porque se reducen costos. También es necesario cumplir con regulaciones para regiones específicas que además sirven como barreras no arancelarias, o ajustarse a normas ambientales, como las ISO14000, que en muchas ocasiones son un requisito para poder integrarse a las cadenas de producción mundial o regional.

A continuación se revisan tres conceptos que se reflejan en la regulación y en la actividad estratégica ambiental de las empresas: el ciclo de materiales de producción y consumo, el diseño de sistemas con múltiples ciclos de vida y el uso de la información para la mejora ambiental.

\subsection{El ciclo de materiales de producción y consumo}

La acumulación de materiales en la biosfera tiene un impacto negativo sobre el ambiente. Para evitarlo se ha propuesto buscar sistemas que permitan cerrar el ciclo de materiales de producción y sistemas de consumo. Esta idea no es nueva, deriva de la observación de los ecosistemas naturales en donde algunos organismos viven de consumir desperdicios que otros producen. ${ }^{5}$ Sin embargo, no se debe perder de vista que la propuesta de una relación simbiótica entre empresas industriales, en la que los desechos de unas son los insumos de otras, tendría riesgos; por ejemplo, si una de ellas sale del mercado, o encuentra más ventajoso no producir el desperdicio o cambiar de producto, se rompería el equilibrio. Ade-

\footnotetext{
${ }^{4}$ Deanna J. Richards, The Industrial Green Game: implications for environmental design and management, National Academy of Engineering, Washington, National Academy Press, 1997, p.1.

${ }^{5}$ Henning Grann, "The Industrial Symbiosis at Kalundborg, Denmark", en The Industrial Green Game: implications for environmental design and management, coord. Deanna Richards, p. 117.
} 
más, la pequeña escala y la diversidad limitada que se podría lograr al organizar industrias por comunidad o región ecológica volverían al pretendido ecosistema industrial muy frágil. ${ }^{6}$

Se debe considerar que cerrar los ciclos de materiales y consumo no es una tarea sencilla, especialmente porque los insumos y desperdicios de la sociedad son compuestos. Es decir, cuando los materiales han sufrido procesos como aleaciones, laminados o recubiertos presentan un reto diferente para su reutilización o reciclaje, ya que en ocasiones los procesos de recuperación resultan altamente contaminantes o no viables económicamente.

En las últimas décadas se han desarrollado tecnologías que ayudan a identificar los distintos materiales y explotar las diferencias en cuanto a propiedades físicas y químicas: el tamaño de partículas y su densidad, así como las propiedades ópticas y magnéticas de los materiales permiten separar la basura orgánica de los metales ferrosos y no ferrosos. Diseñar los productos para facilitar esta discriminación se ha vuelto muy importante porque permite una recuperación más fácil y económica.

Por otro lado, se observa que algunos sistemas industriales han creado de manera natural mercados donde unas empresas consumen los desechos industriales de otras. Basándose en estas experiencias se pueden encontrar formas de incentivar la creación o crecimiento de mercados para uso de materiales reciclados. ${ }^{7}$ El objetivo final para un sistema industrial ecológicamente saludable es que prácticamente todos los materiales que se empleen recorran el ciclo completo una y otra vez.

\subsection{Productos con múltiples ciclos de vida}

En esta propuesta, los productos son usados en varios sistemas o ciclos de producto ya sea como partes, como materiales o como productores de energía. Esencialmente, un producto se convierte en un insumo para varios ciclos en lugar de ser desechado después de su primer ciclo de vida. Esta idea ha sido impulsada por las presiones de regulación (la legislación para recuperar los productos después de su vida útil) y está llevando a las compañías a innovar en

\footnotetext{
${ }^{6}$ Deanna Richards, op. cit., p. 10.

${ }^{7}$ Robert. A. Frosch, "Closing the Loop on Waste Material", en The Industrial Green Game: implications for environmental design and management, p. 38.
} 
diferentes maneras: a) diseño del producto y de la producción, b) selección de materiales, c) administración de la calidad ambiental en la cadena de abastecimiento, d) administración de inventarios y distribución de pedidos, y e) un sistema de información que incluya la visión ambiental. ${ }^{8}$

\section{a) Diseño del producto y de la producción}

El primer paso al diseñar un producto es definirlo determinando los requerimientos del cliente, el costo, la seguridad, la confiabilidad, la facilidad de manufactura y de reciclaje. Al determinar las características del producto se podrán desarrollar diferentes estrategias de materiales y del sistema de producción.

Cuando el negocio permite aprovechar la misma plataforma de producción para varios productos, es mejor que se diseñen para fácil escalamiento y para intercambio de partes, componentes o sistemas entre líneas de productos. Este sistema ofrece oportunidades sustanciales para reducir desperdicios sólidos, pues sólo algunas partes necesitan ser rediseñadas para alcanzar un nuevo modelo y no es necesario desechar herramientas o partes de la plataforma. Por ejemplo, los diseños que facilitan el intercambio de tableros de circuitos permiten escalar los tableros para los nuevos modelos. Características de diseño como éstas — aunadas a actividades de recuperación y remanufactura - pueden resultar en una importante reducción de desechos sólidos.

Otro objetivo al diseñar productos incluye utilizar componentes que usen menos materiales o que sean más eficientes desde el punto de vista del costo energético. La reducción del uso de materiales se traduce fácilmente en menores costos (se compra menos, se usa menos energía en manufactura, hay menos costos de transporte y menos materiales que manejar al final de su vida útil). Un ideal en este sentido es llegar a la transformación de los productos minimizando el uso de materiales, por ejemplo, la digitalización de toda la información.

Al diseñar la producción con un enfoque ambiental, el objetivo es minimizar los residuos generados durante la fabricación del producto. A menudo la estrategia es usar los procesos tecnológicos más modernos porque tienden a ser más eficientes y limpios. En general, se puede decir que hay intercambio entre costo, efectividad e

${ }^{8}$ Deanna Richards, op. cit., p. 12. 
impacto ambiental al seleccionar la tecnología. La calidad ecológica de la fabricación suele medirse por la cantidad de desechos producidos por unidad; en este sentido la disminución de reprocesos y desperdicios impacta de manera favorable al ambiente.

b) La selección de materiales

El uso de materiales es uno de los aspectos más importantes al administrar para la calidad ambiental. La selección de materiales, la evaluación química y el análisis del ciclo de vida son maneras importantes de mejorar la reutilización y reciclaje que pueden disminuir costos o generar utilidades. Cuando los materiales se escogen adecuadamente es más fácil crear relaciones efectivas en costo con los recicladores o vendedores de materiales. La manera más sencilla de hacerlo ha sido la publicación de listas de materiales prohibidos o permitidos. En los últimos años han aparecido métodos mejores y más complicados como el análisis del ciclo de vida del producto.

El análisis del ciclo de vida del producto se ha definido como una forma de evaluar los efectos ambientales asociados a cualquier actividad industrial, desde la reunión inicial de materias primas a partir de su extracción hasta que éstas retornan a la tierra. De igual manera, este análisis intenta evaluar el impacto ambiental del producto en varias etapas, desde la extracción de materiales y producción hasta la distribución y disposición de los mismos; se hace a través de tres procesos sucesivos: ${ }^{9}$

- Primero se hace el inventario de ciclo de vida. Se cuantifican los insumos de energía y materias primas y los resultados del producto, emisiones al aire y agua, desechos sólidos y otras emisiones relacionadas con el ciclo de vida del producto, proceso o actividad.

- Después se analiza el impacto del ciclo de vida. Se hace una caracterización del efecto de la carga ambiental identificada en el inventario del ciclo de vida y una estimación del impacto ecológico y de salud.

- Por último, se mejora el ciclo de vida. Es una evaluación sistemática de las necesidades y oportunidades para reducir la carga ambiental de los insumos y productos que se inventariaron al analizar el ciclo de vida.

9 Ibidem, p.19. 
Se han identificado varios problemas con el análisis del ciclo de vida: es metodológicamente complejo; se deben fijar niveles mínimos de desagregación porque muchos detalles hacen que se pierda lo importante y si está muy agregado produce resultados sin significado; necesita mucha información y recursos, deja de lado factores cualitativos y frecuentemente usa factores subordinados inadecuados para estimar el impacto ambiental. ${ }^{10}$ Sin embargo, realizar el análisis ha demostrado su valor, ya que cuando las empresas se dan cuenta del problema de contaminación se activan para mejorarlo.

c) Administración de la calidad ambiental en la cadena de abastecimiento

Actualmente, han aparecido empresas que operan globalmente y que organizan la producción en cadenas complejas en el nivel mundial; distribuyen partes del proceso productivo en diferentes países donde encuentran ventajas comparativas como acceso a capital humano altamente calificado, mano de obra muy barata, menores costos y acceso a materias primas o a mercados específicos. Estas empresas subcontratan procesos productivos; es decir, la investigación y desarrollo, el diseño y la fabricación de partes y componentes son hechos por diferentes proveedores.

La importancia de evaluar la calidad ambiental de las cadenas de abastecimiento es crítica porque hay estándares y prácticas diferentes. Los proveedores deben cumplir con la regulación ambiental de los países en donde los productos eventualmente van a ser vendidos. Las prácticas de los proveedores deben conformarse a cualquier etiquetado que las compañías usan para designar su producto como amigable con el ambiente. Los fabricantes deben entender y estandarizar muchas de las prácticas de los proveedores para asegurar la calidad ambiental de la cadena de proveeduría usando encuestas, auditorías y certificados expedidos por una tercera parte como son las ISO $14000 .{ }^{11}$

Las empresas y sus proveedores también establecen proyectos conjuntos para lograr metas de reutilización, reciclaje y mejora ambiental; por ejemplo, cuando la sociedad científica se dio cuenta que los clorofluocarbonos que se usaban

\footnotetext{
${ }^{10}$ Robert Johnston "A Critique of Life Cycle Analysis: paper products", en The Industrial Green Game: implications for environmental design and management, p. 226.

${ }^{11}$ Rolf Manstrander, "Hydro Alumninium's Experience with Industrial Ecology", en The Industrial Green Game: implications for environmental design and management, p. 149.
} 
como solventes para limpiar los circuitos electrónicos depletaban el ozono, se solicitó a las compañías productoras de partes electrónicas dejar de usarlo y se desarrolló un sistema basado en agua que fue compartido con la cadena de proveedores. $^{12}$

\section{d) La administración de inventarios y la distribución de pedidos}

La forma en que se surten los pedidos puede tener fuertes implicaciones ambientales, específicamente el empaque, la administración del inventario y la distribución logística. La estrategia para enfrentar los problemas con el empaque es similar a la asociada con el producto: usar menos material, usar material biodegradable y diseñar el empaque para reutilización y reciclaje.

Por otro lado, administrar el inventario y surtir los pedidos requieren de predicciones exactas y del uso de la logística. Como ejemplo de ello tenemos las prácticas de manufactura justo a tiempo que buscan igualar la producción a la demanda del mercado para eliminar almacenaje o excedentes de producto, asimismo reflejan cómo las predicciones acertadas pueden minimizar el almacenaje de productos. Un pronóstico mal hecho y una administración de inventario ineficiente son fuente de costos para la firma y enmascaran fuentes de impacto ambiental. Además, la logística es importante en la distribución por la energía que se utiliza en el transporte; un buen control puede ayudar a disminuir costos y ganancias en calidad ambiental. ${ }^{13}$

e) Un sistema de información que incluya la visión ambiental

Uno de los principales problemas para establecer una política ambiental es el desconocimiento de las consecuencias de las decisiones tomadas por los diferentes actores respecto a temas ambientales. La información es un elemento clave para formar juicios, prever consecuencias y dictar política ambiental. Es esencial contar con un sistema de información que contemple una base de insumos de materiales y energía, descargas de emisiones, referencias para seleccionar los materiales y evaluar el uso de químicos, detalles para administrar la cadena de proveedores, mejorar los controles de inventario y la logística.

\footnotetext{
${ }^{12}$ Deanna Richards, op. cit., p.21.
}

13 Ibid., p. 22. 
En los últimos años se ha planteado la necesidad de que los sistemas de información incluyan contabilidad de costos ambientales, medidas de desempeño ambiental y actitudes del consumidor hacia problemas ambientales. La contabilidad de costos debe identificar cómo es que éstos cambian como resultado de decisiones relacionadas con el ambiente. La medición del desempeño ambiental debe incluir medidas de impacto ambiental y de riesgo o probabilidad de ocurrencia de descargas ambientales inesperadas. La información sobre actitudes de los consumidores debe ayudar a explicar sus expectativas sobre bienes o servicios desde el punto de vista ambiental, el rol que esperan que tengan las empresas, los tipos y niveles de información ambiental que desean conocer y el impacto de la publicidad ambiental. ${ }^{14}$

\subsection{Instrumentos de regulación ambiental}

Los instrumentos de regulación pueden ser directos o indirectos:

Los instrumentos de regulación directa corresponden a medidas institucionales dirigidas a influir directamente el comportamiento ambiental de los actores. Regulan los procesos productivos o los productos, prohíben o limitan las descargas de ciertos contaminantes, y/o restringen actividades en ciertos periodos o áreas. Los instrumentos económicos -o de regulación indirecta - son todos aquellos instrumentos que inciden en los costos y beneficios imputables a los cursos de acción alternativos que enfrentan los agentes económicos. En otras palabras un instrumento económico afecta la rentabilidad de procesos o tecnologías alternativas, o el precio relativo de un producto. El instrumento económico altera las decisiones de productores y consumidores, induciéndolos eventualmente a acciones que en el conjunto contribuirán a reducir los niveles de deterioro ambiental. ${ }^{15}$

Las prohibiciones e impuestos son herramientas no regulatorias basadas en el mercado que se usan para reducir el costo ambiental asociado con los materiales; los impuestos desaceleran el uso de sustancias o productos no deseables.

Cada instrumento tiene un ámbito particular de aplicación y diferentes condiciones de alcance. Las normas y su verificación coactiva son instrumentos aptos para ser aplicados en procesos, productos o actividades estandarizadas y alcanzan su

\footnotetext{
14 Ibid., p. 24.

${ }^{15}$ Secretaría del Medio Ambiente y Recursos Naturales (SEMARNAT), Instrumentos económicos para la gestión ambiental en América Latina y el Caribe, México, 1998, pp. 18-19.
} 
mejor desempeño cuando su cobertura es general y su contenido fácil de entender, aplicar y vigilar. Otros instrumentos tienen mayor especificidad y sólo pueden imponerse a actividades claramente determinadas en el tiempo y el espacio. ${ }^{16}$

Hay otras fuerzas no regulatorias que son parte del juego ambiental actual, como los estándares ambientales voluntarios de la International Organization for Standarization (ISO-14000). La serie de normas ISO 14000 es una iniciativa en materia de administración del medio ambiente que se registra dentro de la tendencia mundial de autorregulación de la industria. Esta serie consta de dos grupos de normas: las dirigidas a la evaluación de una empresa y la dirigida a etiquetado ecológico y ciclo de vida de un producto. La adopción de la norma es voluntaria, pero necesita estar certificada por un organismo privado. ${ }^{17}$

Las instituciones ambientales han puesto énfasis en cómo las empresas deben abordar los problemas ambientales que afectan la salud y la seguridad: cerrar el ciclo de materiales de producción y consumo, diseñar sistemas con múltiples ciclos de vida y el uso de la información para la mejora ambiental se empiezan a reflejar en la regulación. Por ejemplo, hay requerimientos legales que están obligando a las empresas a recolectar sus productos al llegar al final de su vida útil y reciclarlos o reutilizarlos en porcentajes determinados. Debido a que la regulación en ocasiones inhibe la innovación, se ha tratado de establecer proyectos de cooperación entre las empresas e instituciones ambientales, así como de establecer estándares de desempeño que las compañías puedan cumplir en diferentes formas.

\section{La Unión Europea y la Directiva 2000/54/EC sobre vehículos que llegan al final de su vida útil}

A finales de la década de los ochenta se empezó a formular una estrategia general para la administración de desechos en los países miembros de la Comunidad Económica Europea, pero la problemática del reciclaje de los ELV se incluyó hasta 1991. La principal preocupación era el residuo automotor por ser de alta toxicidad y arrojarse a los tiraderos como relleno sanitario. Este residuo representa el 24\% del peso de un automóvil y está compuesto de plásticos, líquidos, hules, vidrios y otras sustancias; el 76\% restante, por hierro y acero. Puede reciclarse fácilmente

\footnotetext{
${ }^{16}$ SEMARNAT, Sistema integrado de Regulación Directa y Gestión Ambiental de la Industria, México, 1997, p. 2.

${ }^{17}$ Ibid., p. 27.
} 
y existen mercados establecidos que usan la chatarra metálica como insumo. ${ }^{18} \mathrm{El}$ reciclaje de vehículos automotores no es un negocio con mucho margen de utilidad; cubre más bien la necesidad de tener un ambiente limpio y sano. No existen mercados importantes de materiales reciclados, lo que detiene la inversión en el desarrollo de la tecnología necesaria para optimizar la industria de reciclaje.

En 1997, después de varios estudios, se presentó una propuesta de Directiva sobre ELV a la Comisión Europea de Administración de Desechos; esta propuesta retomaba algunos acuerdos y leyes alemanas que requerían a todos los fabricantes de automóviles participar activamente en el reciclamiento de las unidades y hacerse responsables de los vehículos de desecho que habían vendido alguna vez en el país. ${ }^{19}$ Los fabricantes de automóviles discutieron durante mucho tiempo respecto a sus obligaciones y plantearon que su papel debía limitarse a fabricar automóviles reciclables que fueran fácilmente desmantelados y no querían pagar por la recuperación y reciclamiento de los vehículos que ya se encontraban en el mercado. ${ }^{20}$

Después de tres años de discusiones, el Parlamento de la Unión Europea aprobó la Directiva 2000/54/EC sobre ELV, que intenta prevenir la acumulación de vehículos de desecho y promover la recuperación, reutilización y reciclaje de sus componentes para proteger el ambiente. La Directiva define un ELV como un vehículo automotor que llega al final de su vida útil e incluye vehículos de cuatro, tres y dos ruedas, además de sus componentes; propone medidas dirigidas a la prevención de desechos, reutilización, reciclaje y otras formas de recuperación de los ELV y sus componentes; asimismo, busca mejorar el desempeño ambiental de los actores económicos involucrados en el ciclo de vida y en operaciones directamente relacionadas con el tratamiento de los vehículos de desecho.A continuación se describen algunos puntos importantes de la Directiva: ${ }^{21}$

- El parlamento considera que las medidas de los diferentes miembros estados de la Unión Europea deben armonizarse para minimizar el impacto de los ELV

\footnotetext{
${ }_{18}$ Tristán Honeywill, “A final bill to pick up”, Professional Engineering, marzo, 2002, p. 28.

${ }^{19}$ Roberto Zoboli y colabs., "Regulation and innovation in the area of end-of.life-vehicles", en The Impact of European Union Regulation on innovation of European Industry, R. Leone (ed), Joint Research Center y el Institute for Prospective of Technological Studies, marzo, 2000, EUR-19598EN, pp. 1.45-1.47.

20 Tristán Honeywill, op. cit., p. 28.

${ }^{21}$ European Parliament and Council of the European Union, "Directive 2000/53/EC of the European Parliament and the Council of 18 September 2000 on end-of-life vehicles", Official Journal of the European Communities, 21-10-2000 pp. L 269/34 - L 269/42.
} 
en el ambiente del continente. Deben contribuir a proteger, preservar y mejorar la calidad ambiental conservar la energía, asegurar las operaciones del mercado y evitar distorsiones por competencia dentro de la Unión Europea.

- Cada uno de los países miembros de la Unión Europea tendrá que generar su propia legislación y podrá alcanzar las metas señaladas mediante acuerdos con los actores económicos.

- La fecha límite de implantación por los estados miembros fue en abril de 2002. ${ }^{22}$

- Los miembros estados asegurarán que el último dueño entregue el ELV a una planta de reciclamiento autorizada sin ningún costo, aun cuando el vehículo tenga un valor de mercado negativo.

- Las empresas fabricantes redujeron el uso de sustancias peligrosas y garantizaron que los componentes de los vehículos puestos en el mercado después del primero de julio de 2003 no contendrán mercurio, cromo, cadmio o plomo, excepto los casos listados en el anexo 1 de la Directiva.

- El almacenamiento y tratamiento de un ELV debe estar sujeto a estricto control y seguir los requerimientos de la Directiva 75/442/EEC sobre disposición de desechos. Se establecerá un sistema para recolectar los vehículos y sus partes usadas, transportándolos a plantas autorizadas para su tratamiento y creando un sistema para darlos de baja después de elaborar un certificado de destrucción.

- Se establecerán estándares de codificación para asegurarse de que los productores usen material con códigos que permitan la identificación de cada material al desmantelar el vehículo.

- La red de empresas recicladoras debe operar conforme a las leyes ambientales y certificarse.

- Es necesario desarrollar procedimientos para informar y educar a los compradores de vehículos sobre la recuperación y reciclamiento de los componentes y el tratamiento de ELV.

En la industria automotriz, las innovaciones impulsadas por nuevas reglas ambientales tienen una larga historia; por ejemplo, la disminución de emisiones contaminantes está relacionada con el uso de nuevos materiales para fabricar automóviles

\footnotetext{
${ }^{2}$ En febrero de 2003 únicamente Austria, Dinamarca, Alemania, Suecia y Holanda habían tomado iniciativas legales para aplicar la directiva. Sistema de monitoreo oficial ACEA, http: //www.acea.be, mayo de 2003 .
} 
más ligeros que consumen menos combustible y que se impulsó en la década de los setenta, después de la crisis del petróleo.

Las innovaciones relacionadas con el reciclaje de vehículos se inician desde los años noventa cuando se inicia la discusión en el parlamento europeo sobre los desechos de la industria de reciclaje. Cuando se decidió regular los ELV en el año 2000 ya se habían realizado muchos proyectos de investigación, se había hecho cabildeo para establecer medidas convenientes de reciclaje y se había implementado el diseño para reciclar y desensamblar en algunas empresas de la industria automotriz. Sin embargo, las innovaciones organizativas en la industria de reciclaje a partir de la aprobación de la directiva son más notorias. A continuación se refirieren innovaciones en el diseño del producto y la producción.

\subsection{Innovación en el diseño para reciclar y desensamblar}

Al diseñar para reciclar se han observado las siguientes acciones.23

a) Se establece el ciclo de vida de producto y su impacto ambiental.

b) Se calculan coeficientes de reciclabilidad de cada vehículo; el coeficiente indica qué porcentaje del vehículo es reciclable y se establecen metas anuales para mejorarlo.

c) La selección de materiales ha sido siempre un proceso complicado. En años previos para el paradigma ambiental los principales criterios eran costos, facilidad de manufactura y disponibilidad de materiales. Actualmente, se revisan las listas de materiales y se hacen estudios de sustitución de los mismos, privilegiándose aquéllos con menos polímeros o seleccionándose aquéllos con más posibilidades de reciclamiento, como el aluminio.

d) Se desarrollan listas de materiales y sustancias no admitidas como parte de las nuevas especificaciones técnicas para los proveedores, lográndose la transferencia de responsabilidades entre industrias. Por esto se dice que el diseñar para desensamblar y diseñar para reciclar tiene aspectos sistémicos.

\footnotetext{
${ }^{23}$ Barbiroli Giancarlo, "Regulation and innovation in the area of end-of life-vehicles", en The Impact of European Union Regulation on innovation of European Industry, R. Leone (ed), volume II, Joint Research Center y el Institute for Prospective of Technological Studies, marzo, 2000, EUR-19598EN, pp. 33-145.
} 
e) Se establecen sistemas de códigos para cada parte del vehículo elaborándose manuales de reciclamiento internos y otros que se entregan a la red de empresas recicladoras.

f) Algunas partes de los nuevos vehículos se empiezan a manufacturar con plástico reciclado como los conductos de aire y de cables.

g) La manufactura flexible también permite la fácil reconfiguración de un vehículo. Diferentes tipos de arquitectura pueden construirse con nodos comunes y tubos extras unidos con métodos eficientes que necesitan baja energía. Esto permite la reutilización de componentes o módulos.

h) Los fabricantes de automóviles están llevando a cabo una gran gama de proyectos de investigación para desarrollar materiales amigables con el medio ambiente y ahorrar energía. Por ejemplo, se busca reemplazar materiales basados en petróleo por materiales basados en vegetales. El poliéster ecoefectivo es una tela que puede ser reciclada y reprocesada en fibra material una y otra vez sin perder cualidades de desempeño; este poliéster puede ser usado en asientos, tablero, volante, interior de las puertas y cabeceras. Otro ejemplo es el polylactaide que es un biopolímero derivado de maíz y que se usará para toldos y tapetes de alfombra. Se está trabajando sobre llantas de hule con rellenos basados en maíz, como un sustituto parcial de negro de humo, que ofrecen menor resistencia y menor peso, lo que puede mejorar la economía y mejorar la tracción en pavimento húmedo.

i) Se ha buscado que los contenedores de fluidos o sustancias dañinas se puedan retirar fácilmente del vehículo, así como las baterías y las bolsas de aire.

j) Se empiezan a usar materiales con memoria (plásticos o aleaciones) que pueden regresar a su forma líquida al calentarse. Esto facilita el desensamble. ${ }^{24}$

k) Se elaboran manuales de desensamble de uso interno para distribuirlos y capacitar a los operarios de las redes de reciclamiento, logrando así transferencia de conocimientos.

\subsection{Patrones de innovación organizativa en la industria de reciclaje}

Respecto al desarrollo de la estructura y de los procesos de la industria de reciclaje se han observado dos patrones: la organización, en algunos de los países, de una red de reciclamiento por los fabricantes de automóviles y el número de empresas en la industria.

${ }^{24}$ Rob Coppinger, "Breaking up is easy to do", en The engineer, 13/26, septiembre de 2002, p. 15. 
Para formar la red de reciclamiento, los fabricantes de automóviles firmaron un acuerdo con empresas desmanteladoras y trituradoras que aceptan los ELV y así tienen gran ingerencia sobre la cadena de reciclaje. Con base en los acuerdos, se crearon empresas que administran el proceso y se encargan de seleccionar y organizar a los diferentes actores — las ensambladoras, los distribuidores de automóviles, los desmanteladores y los trituradores. El procedimiento establecido es el siguiente: los distribuidores recogen los ELV del último dueño, se elabora un certificado y se preparan los vehículos para ser entregados al centro de reciclado. Los centros de reciclado son una red de empresas con tecnologías modernas que cumplen los estándares ambientales y están certificados. El centro recibe el vehículo a precio de mercado y desempeña el tratamiento especificado. El chasis del vehículo se envía a una empresa recicladora de hierro y acero mientras que el resto se envía a un centro de triturado certificado y miembro de la red. A su vez, los trituradores tienen la obligación de disponer del residuo que debe incinerarse o llevarse a tiraderos, donde operadores seleccionados cumplen con la regulación. Existe un sistema de logística que asegura el control y detección inmediata de los vehículos de desecho y de materiales; se han desarrollado sistemas de información y redes de cooperación entre fabricantes de automóviles. ${ }^{25}$

Los centros de recolección tienden a crecer en número, mientras que los centros de triturado tienden a disminuir; esto está relacionado con el proceso de certificación. Los desmanteladores con licencia han pasado de 7,303 a 9,576 entre 1989 y 2003 mientras que los centros de triturado pasaron de 222 a 158 en el mismo periodo. ${ }^{26}$

Las ventajas de la asociación entre fabricantes de vehículos y recicladores que se han observado son las siguientes: información sobre aspectos legales, financiamiento de equipos para desmantelamiento, asesoramiento sobre el mercado de partes usadas, sistemas de información en línea para desmantelar cada modelo y capacitación a las empresas recicladoras.

${ }^{25}$ Zoboli, op. cit., pp. III.1-III.11

${ }^{26}$ Zoboli y colabs., op. cit., p. 1.34; Sistema de monitoreo ACEA, http://www.acea.be, mayo de 2003, p. 6. 
Política ambiental y su impacto en la innovación tecnológica

y organizativa: el reciclaje de vehículos automotores

\section{Cuadro 1 \\ Actividades implantadas por las empresas fabricantes de automóviles para cumplir con la Directiva 2000/54/EC de la Unión Europea}

\begin{tabular}{|c|c|}
\hline Empresa & Acciones \\
\hline $\begin{array}{l}\text { Renault, } \\
\text { S.A. }\end{array}$ & $\begin{array}{l}\text { Recolección de automóviles, reciclaje de materiales, recuperación de energía } \\
\text { Investigación y desarrollo en reciclamiento de plástico } \\
\text { Contratos con } 270 \text { empresas desmanteladoras } \\
\text { Promedio de reuso recuperación y reciclamiento (rrr) de } 82.9 \% \text { en los vehículos } \\
\text { Renault }\end{array}$ \\
\hline $\begin{array}{l}\text { PSA Peugeot } \\
\text { CITROEN }\end{array}$ & $\begin{array}{l}\text { Diseño de vehículos para que sean } 90 \% \text { reciclables a partir de } 2002 \\
\text { Reciclamiento y reutilización de partes } \\
\text { Reducción de diversidad de materiales } \\
\text { Mayor uso de materiales reciclables en nuevos automóviles }\end{array}$ \\
\hline $\begin{array}{ll}\text { Adam } & \text { Opel } \\
\text { AG } & \end{array}$ & $\begin{array}{l}\text { Recuperación de vehículos y diseño para mejorar reciclamiento } \\
\text { Red de } 234 \text { empresas desmanteladoras } \\
\text { Manuales para uso interno de diseño para reciclar } \\
\text { Coeficientes de reciclamiento calculados para uso interno } \\
\text { Análisis de ciclo de vida para materiales y componentes }\end{array}$ \\
\hline BMW & $\begin{array}{l}\text { Recuperación de vehículos y diseño para mejorar reciclamiento } \\
\text { Red de } 90 \text { empresas desmanteladotas asociadas } \\
\text { Diseño para reciclar, desmantelar y coeficientes de reciclaje en componentes y } \\
\text { partes }\end{array}$ \\
\hline $\begin{array}{l}\text { Daimler } \\
\text { Chrysler }\end{array}$ & $\begin{array}{l}\text { Diseño para reciclar y diseño para desmantelar } \\
\text { Simplificación de régimen de materiales al reducir el número de piezas de plástico } \\
\text { El ciclo de vida del producto se analiza para evaluar las alternativas de materiales }\end{array}$ \\
\hline $\begin{array}{l}\text { Ford } \\
\text { Motor } \\
\text { Company }\end{array}$ & $\begin{array}{l}\text { Restricciones en el uso de sustancias peligrosas y guías para diseño para reciclar } \\
\text { Estándares de codificación en fabricación de partes y materiales } \\
\text { Metas de reciclamiento en los nuevos modelos y uso de materiales reciclables } \\
\text { Red de } 180 \text { empresas desmanteladoras en Alemania } \\
\text { El ciclo de vida del producto se usa para seleccionar materiales y componentes }\end{array}$ \\
\hline FIAT & $\begin{array}{l}\text { Sistemas efectivos de desmantelamiento, reuso de materiales reciclables. } \\
\text { Recuperación de energía del material desmenuzado } \\
\text { Red de } 312 \text { empresas desmanteladoras asociadas } \\
\text { Tasa de recuperación se calcula a } 82 \% \text { del peso del vehículo } \\
\text { Coeficientes de reciclamiento para uso interno } \\
\text { Análisis de ciclo de vida aplicado a materiales y componentes, por ejemplo, } \\
\text { acuerdos con Rhone Poulec para reciclar convertidores catalíticos y } \\
\text { recuperar los metales preciosos como son platino, rodio, palladium }\end{array}$ \\
\hline $\begin{array}{l}\text { Volvo Car } \\
\text { corporation }\end{array}$ & $\begin{array}{l}\text { Diseño para desmantelar, reciclar y recuperación de energía del material } \\
\text { desmenuzado } \\
\text { Cooperación en reciclamiento con proveedores de materiales y componentes } \\
\text { Red de } 70 \text { empresas desmanteladotas en Suecia }\end{array}$ \\
\hline
\end{tabular}

Fuente: M. Mazzanti, y R. Zoboli, "Environmental policy instruments, cost-minimising strategies, and induced innovation. The case of end-of- life Vehicles". Documentos de trabajo 


\subsection{Los mercados para productos reciclados}

Se pueden identificar tres posibles trayectorias de innovación respecto a la creación de mercados: la creación de un mercado de materiales, la creación de un mercado de energía y la trayectoria de sustitución radical. ${ }^{27}$

Aumentar el porcentaje de materiales reciclados implica crear un mercado que los consuma. Específicamente, en el caso del plástico se requerirían innovaciones en su uso, ya sea en la industria automotriz o en otras industrias. Además, puede suceder que al aumentar la cantidad de plástico reciclado las fuentes de materia prima virgen sufren pérdidas de mercado. La creación de un mercado de materiales significa la adecuación económica entre materia prima virgen, reciclada y necesidades de mercado. Además, es muy importante obtener un flujo de materiales reciclados en cantidad, calidad y costo constantes que permitan el crecimiento de la industria.

El número de empresas que pueden incinerar el material triturado y aprovecharlo como energía es aún muy limitada, por lo que esta trayectoria debe incluir nuevas relaciones entre empresas trituradoras e industrias que consumen energía $u$ otros sectores que usen combustible derivado de desperdicios como la industria del cemento. El potencial de reciclaje depende en gran medida de la presencia de residuos de polímeros y la retroalimentación en diseño y mezcla de materiales; asimismo, las emisiones al ambiente deben ser evaluadas y comparadas con el uso de fuentes de energía convencional.

La sustitución radical de materiales puede darse por las dificultades de crear mercados de materiales o de energía. Las restricciones en el uso de materiales pueden interrumpir la tendencia a usar componentes de materiales basados en polímeros; además, si es viable económicamente usar ecopolímeros, poliéster ecoefectivo u otros tipos de materiales ecológicos, los nuevos automóviles utilizarán estos materiales mientras no afecten su desempeño.

\section{El reciclaje de vehículos automotores en México}

En México, la política ambiental tomó importancia a partir de los años setenta y ha evolucionado dispersa y contradictoriamente porque las funciones de protección

\footnotetext{
27 Ibidem.
} 
ambiental se encontraban divididas en varias instituciones. Aunado a esto, la falta de experiencia en este campo llevó a utilizar lineamientos de diversos organismos internacionales que no consideraban las circunstancias nacionales en forma integral.

La solución a problemas ambientales ofrece oportunidades para la inversión productiva y la creación de fuentes de empleo. Sin embargo, el sector privado ha limitado su participación y el crecimiento de la población - combinado con la situación de pobreza - han llevado a la destrucción de recursos naturales. ${ }^{28}$

Una política ambiental requiere de la comprensión y reconocimiento pleno de un problema por parte de la sociedad y del gobierno. También se requiere que las autoridades demuestren tener capacidad técnica y de convocatoria entre los diferentes actores para poder instrumentar soluciones. El gobierno mexicano ha carecido de estos requisitos y en una situación económica precaria es difícil dirigir recursos para resolver problemas ambientales. ${ }^{29}$

Situaciones como éstas llevan a que los sistemas de información y vigilancia de las leyes ambientales sean limitados. En 1995 se planteó la necesidad de un sistema nacional de información ambiental que incluyera un inventario de emisiones y transferencia de contaminantes, un sistema de información de cumplimiento de normatividad y una cédula de desempeño ambiental por establecimiento, que aún no se ha conformado como tal. ${ }^{30}$

En este marco de referencia encontramos que el Tratado de Libre Comercio para América del Norte establece que a partir del 2009 se podrán importar de Estados Unidos y Canadá vehículos con diez años de antigüedad o más y la permisividad de antigüedad disminuirá progresivamente hasta que en el 2019 se pueda importar todo tipo de vehículos. ${ }^{31}$ Esto representará un incremento exponencial del parque vehicular en México y graves problemas de reciclamiento de los ELV.

\footnotetext{
${ }^{28}$ Roberto Escalante, Instrumentos económicos para la gestión ambiental: los aceites lubricantes usados en México, México, Facultad de Economía, UNAM, DGAPA, México, p. 16.

${ }^{29}$ Ibid., p. 23.

${ }^{30}$ SEMARNAT, Sistema integrado de Regulación Directa y Gestión Ambiental de la Industria, México, 1997, pp. 24 y 25.

${ }^{31}$ Secretaría de Comercio y Fomento Industrial,"Decreto de Promulgación del Tratado de Libre Comercio de América del Norte", Diario Oficial de la Federación, 20 de diciembre de 1993, p.45.
} 
Las autoridades ambientales en México no contemplan el problema de los ELV de una forma integral. Existe regulación sobre desechos peligrosos, baterías, aceites y lubricantes. Ha habido propuestas de las autoridades ambientales para establecer sistemas de depósito y reembolso para baterías de automóviles, neumáticos, lubricantes y baterías para aparatos eléctricos; sin embargo, existen muchos problemas para que las empresas las acepten. Por ejemplo, los fabricantes de llantas del país pusieron como condición que se prohibiera la importación de neumáticos usados en la frontera norte; la asociación de la industria química señaló que el sistema para lubricantes representa costos y que es más barato el aceite virgen que el reciclado para la elaboración de lubricantes; por su parte, el sistema de pilas y baterías no ha mostrado su viabilidad económica y no se tienen los estudios, por lo que tampoco se ha implementado. El reciclaje de baterías de automóviles es un sistema de depósito reembolso que funciona desde hace muchos años y se calcula que el $75 \%$ de las baterías de desecho se reciclan. ${ }^{32}$

\subsection{Problemas de contaminación por vehículos y sus partes}

En México ya existen problemas graves de contaminación por vehículos y sus partes, especialmente en la frontera norte entre los cuales destacan los grandes tiraderos de llantas, los terrenos llenos de automóviles inservibles y la contaminación del subsuelo por aceites y anticongelantes.

Los tiraderos de llantas son insalubres y ocasionan la aparición de mosquitos, que pueden producir enfermedades como el dengue; hay plaga de ratas y serpientes; además, esos depósitos pueden incendiarse y causar graves daños al ecosistema.

El año pasado en los Estados Unidos se desecharon 280 millones de llantas de las cuales se recicla el $80 \%{ }^{33}$; las 56 millones restantes se distribuyen entre tiraderos de aquel país y México. Las llantas de medio uso se venden junto con las inservibles en grandes lotes que se distribuyen en el norte México, mientras que otras son recolectadas por los tire jockeys y depositadas ilegalmente en tiraderos de ciudades fronterizas como Ciudad Juárez, Reynosa y Laredo donde se reportan pilas con millones de llantas de desecho. A pesar de que se ha comunicado que empresas de la ciudad de Eagle Pass en Texas invertirán 200 millones de dólares para la

\footnotetext{
32 Roberto Escalante, op. cit., pp. 74-78.

${ }^{33}$ Environmental Protection Agency. http://www.epa.gov/cgi-bin/epaprintonly.cgi, 6 de febrero de 2004.
} 
creación de cinco plantas recicladoras de llantas en México, no se ha firmado ningún acuerdo sobre este problema. ${ }^{34}$

Los yonkes o deshuesaderos también han proliferado. No se tienen datos exactos de cuántos son o de cuántos vehículos existen o se desmantelan en estos lugares. Se nutren de manera natural por la actividad de individuos que atraviesan la frontera y compran vehículos usados a precios muy bajos. Los autos usados se utilizan un tiempo, generalmente en la zona fronteriza, y cuando se descomponen no existen piezas de repuesto y es más caro repararlos que venderlos en un deshuesadero o abandonarlos para comprar otro automóvil usado en los Estados Unidos. Al respecto, se ha observado un flujo de partes metálicas fácilmente reciclables, como puertas limpias, de las ciudades fronterizas hacia empresas recicladoras de ese país. Es preocupante que se exporten las partes metálicas y se queden los residuos contaminantes de los ELV, especialmente porque México es un país que importa chatarra metálica que se consume en industrias como la siderúrgica.

En México se generan alrededor de ocho millones de toneladas de residuos peligrosos al año y sólo el 10\% tiene un tratamiento adecuado. ${ }^{35}$ Reciclar aceites y lubricantes es un problema urgente que aún no tiene propuestas viables de solución. Si bien ya se han desarrollado leyes e instrumentos económicos, falta la infraestructura y la participación de los empresarios.

Actualmente, no existen registros exactos del parque vehicular y su antigüedad. Esto se debe a que hay muchos vehículos que entran y salen del país ilegalmente y no se pueden considerar en las estadísticas. La Secretaría de Comunicaciones y Transportes reportó 17.7 millones de vehículos en 2001 distribuidos de la siguiente manera: 12'185,317 de automóviles, 222,259 camiones de pasajeros y 5'376, 870 camiones de carga ${ }^{36}$ De este total 7 millones tienen más de 10 años y por lo menos una cuarta parte pueden ser candidatos a reciclarse; además, se considera que en el país hay aproximadamente 2 millones de vehículos ilegales procedentes de los Estados Unidos. En México no existe la cultura de reciclaje y no se contempla el reciclaje profesional de estos vehículos; muchos de ellos terminan en deshuesaderos y se venden como partes usadas, otros tantos únicamente se amontonan en terrenos baldíos.

\footnotetext{
${ }^{34}$ http://noticias.vanguardia.com.mx, 25 de febrero de 2004.

${ }^{35}$ Escalante, op. cit. pp. 9.

${ }^{36}$ INEGI, Anuario Estadístico de los Estados Unidos Mexicanos, 1998 y 1999, Secretaría de Comunicaciones y Transportes, Dirección General de Planeación, México, 2002. cuadro 8.7.1.1
} 
Los pocos vehículos que se reciclan en México provienen principalmente de la Secretaría de Hacienda y Crédito Público, de la Procuraduría General de Justicia y de las aduanas de Chihuahua, Laredo, Reynosa, Tijuana y Cd. Juárez. Estos vehículos se destruyen porque ingresaron ilegalmente al país o fueron decomisados, no porque lleguen al final de su vida útil. También se reciclan vehículos de programas promovidos por el gobierno para renovar el transporte público, pero la cantidad es mínima. ${ }^{37}$

Entre los problemas que se tienen para reciclar los vehículos decomisados se encuentra la regulación que no permiten tener acceso inmediato. En primer lugar hay que anunciarlos en el Diario Oficial de la Federación y esperar 90 días hábiles a que no haya reclamaciones. Después hay que recoger los vehículos de los "cementerios" donde a veces permanecen por años y son saqueados. No se destinan recursos para cuidarlos porque se van a destruir. Finalmente, cuando llega el momento de destruirlos la policía debe custodiar a los recicladores mientras recogen y aplastan los vehículos porque muchos son adoptados por personas sin hogar para vivir en ellos y reaccionan violentamente cuando les quitan los vehículos. ${ }^{38}$

En México existen aproximadamente 2000 pequeños recicladores, principalmente dedicados al reciclaje de metales, vidrio y papel. ${ }^{39}$ Muchos no se encuentran registrados y aparecen y desaparecen fácilmente. Una de las plantas más grandes equipada ex profeso para reciclar automóviles es CFF Recycling México. La planta inició operaciones en enero de 1998, en ese entonces estimó que existían 750 mil vehículos decomisados que podrían destruir; sin embargo, no ha podido tener acceso a estos vehículos. La falta de cultura de reciclaje de vehículos, las leyes que retardan el proceso de destrucción de los vehículos decomisados y el sistema de recaudación de impuestos que la hace perder oportunidades para adquirir materia prima de los pequeños recolectores dificultan la obtención de materia prima para operar. ${ }^{40}$ La planta puede procesar 192 vehículos por día o 70 mil al año, pero el $97 \%$ de lo que procesa es chatarra doméstica: muebles metálicos, línea blanca, electrónicos, etcétera.

\footnotetext{
${ }^{37}$ Entrevista realizada a la ingeniera. Ma. Elena Cantú, Directora de CFF Recycling México, en noviembre de 2003.

${ }^{38}$ Idem.

${ }^{39}$ Instituto Nacional de Reciclaje (INARE).

${ }^{40}$ Ma. Elena Cantú, op. cit.
} 
Éstos son algunos de los problemas que se presentan para el reciclaje de vehículos automotores en México: falta de cultura de reciclaje, falta de recursos económicos para sustituir el parque vehicular antiguo, falta de sistemas de información confiables y procedimientos inadecuados que retardan los procesos de reciclaje.

\section{Conclusiones}

En el caso estudiado de los ELV se puede observar el aspecto sistémico de los cambios impulsados por la política ambiental. El diseño para desensamblar y reciclar afecta a las empresas fabricantes de vehículos, a sus proveedores y a los productores de materiales.

$\mathrm{Al}$ interior de las empresas, el diseño para desensamblar y reciclar afecta el rumbo de los proyectos de investigación y lleva a innovaciones en el proceso y en el producto.

Al crear un sistema estandarizado de codificación de materiales, se modifica la organización interna y la de proveedores; además, los materiales prohibidos afectan los proceso de compra y producción de las empresas.

Indudablemente, se debe poner cuidado en desmantelar y reciclar materiales que no justifican su costo. La creación de mercados que utilicen productos reciclados es sumamente importante para incentivar el reciclaje. De igual forma, se observa la capacidad de convocatoria y organización de las empresas fabricantes de automóviles para reorganizar la industria recicladora y mantener nuevas relaciones de asociación y cooperación entre ellas. Esta organización les ha permitido mantener el constante flujo de materiales, lo que es muy importante para lograr utilidades e incentivar la creación o crecimiento del mercado de materiales reciclados. En México no existen leyes ni instrumentos específicos que consideren el reciclaje de vehículos automotores de forma integral. El problema se empieza a reconocer, pero no se tienen confiables sistemas de información que permitan analizar todas las aristas.

Las autoridades ambientales ven el problema de los ELV de una forma fragmentada. Se regulan sustancias peligrosas aunque — como se mencionó- no existe la infraestructura para disponer de estos desechos adecuadamente. El problema de las llantas se reconoce, sin embargo no es atendido adecuadamente y no se han podido establecer negociaciones bilaterales. 
Debido a los niveles de pobreza que hay en el país, muchos de los mexicanos no tienen un vehículo en el transcurso de su vida. Una alternativa son los baratos automóviles y camionetas que cruzan la frontera en forma ilegal, lo que representa tanto problemas como oportunidades.

El reciclaje de los ELV puede verse como una oportunidad para incentivar el crecimiento de varias industrias. La industria de reciclaje se puede beneficiar de inversiones que creen empleo y ayuden a mantener la calidad ambiental. El reto está en que la sociedad civil, las empresas, especialmente las de la industria automotriz, y el gobierno mexicano y sus socios en el TLC cooperen para crear la regulación y acuerdos necesarios para aprovechar esta oportunidad y cuidar el medio ambiente.

\section{Bibliografía}

Bellmann, Klaus y Anshuman Kare, Economic issues in recycling en-of-life vehcles, Technovation, no. 20, 2000, pp. 677-690.

Barbiroli, Giancarlo, "Regulation and innovation in the area of end-of. lifevehicles" volume II, R. Leone (ed), The Impact of European Union Regulation on innovation of European Industry, Joint Research Center y el Institute for Prospective of Technological Studies, marzo, 2000, EUR19598EN, 174 pp.

Escalante, I. Roberto y Fidel Aroche, Instrumentos económicos para la gestión ambiental: el caso de los aceites lubricantes en México, Facultad de Economía, DGAPA, México, 2003,219 pp.

European Parliament and Council of the European Union, "Directive 2000/53/EC of the European Parliament and the Council of 18 September 2000 on end-oflife vehicles", Official journal of the European Communities, 21-10-2000 pp. L 269/34 - L 269/42.

Frosch , Robert. A., "Closing the Loop on Waste Material", en The Industrial Green Game: implications for environmental design and management, coord. Deanna Richards, NationalAcademy of Engineering, Washington, National Academy Press, 1997, 280 pp. 
Goodfelow, Melanie, EU's End-of-life-vehicle: rules stuck in neutral, Environmental Finance, abril de 2002.

Henning, Grann, "The Industrial Symbiosos al Kalundborg, Denmark", en The Industrial Green Game: implications for environmental design and management, coord. Deanna Richards, NationalAcademy of Engineering, Washington, National Academy Press, 1997, 280 pp.

INEGI, Anuario Estadístico de los Estados Unidos Mexicanos, 1998 y 1999, Secretaría de Comunicaciones y Transportes, Dirección General de Planeación, México, 2002. cuadro 8.7.1.1

Johnston, Robert "A Critique of Life Cycle Analysis: paper products", en The Industrial Green Game: implications for environmental design and management, coord. Deanna Richards, NationalAcademy of Engineering, Washington, National Academy Press, 1997, 280 pp.

Massanti, Massimiliano y Roberto Zoboli, "Environmental policy instruments, cost minimizing strategies, and induced innovation: The case of end of life vehicles" Documentos de trabajo, IDSE-CNR, Research Institute on the Dynamicas of Economic Systems, National research Council of Italy.

Manstrander, Rolf, "Hydro Aluminium's Experience with Industrial Ecology", en The Industrial Green Game: implications for environmental design and management, coord. Deanna Richards, NationalAcademy of Engineering, Washington, National Academy Press, 1997, 280 pp.

Richards, Deanna J., en The Industrial Green Game: implications for environmental design and management, National Academy Press, Washington, $1997,280 \mathrm{pp}$.

Secretaría de Comercio y Fomento Industrial (SECOFI), "Decreto de Promulgación del Tratado de Libre Comercio de América del Norte", Diario Oficial de la Federación, 20 de diciembre de 1993.

Secretaría del Medio Ambiente Recursos Naturales y Pesca (SEMARNAT), Sistema Integrado de Regulación Directa y Gestión Ambiental de la industria en México, México, 1997, 172 pp. 
Secretaría del Medio Ambiente y Recursos Naturales y Pesca (SEMARNAT), Instrumentos Económicos para la Gestión Ambiental en América Latina y el Caribe, México, 1998, 121 pp.

Zoboli, Robert y colabs., "Regulation and innovation in the Area of End-of-life Vehicles", The impact of European Union regulation on innovation of European Industry, F. Leone (ed), Joint Research Center (JRC) and Institute for Prosepective of Technological Studies (IPTS) EUR 19598, marzo 2000, III.47 pp.

Association des Constructeurs Europeen d' automóbiles (ACEA), "Sistema de monitoreo", mayo, 2003, pp. 1-6, http://www.acea.be

Burger, Marton y Dan Nashaat, "Car recycling rule to cost billions", Business Journal, Budapest, abril, 22/28, 2002, http://www.bbj.hu

Environmental ProtecctionAgency, 6 de febrero de 2004, http:/www.epa.gov/cgibin/epaprintonly.cgi

Honeywell, Tristan, "A final bill to pick up", Professional Engineering, 27 de marzo de 2002, p.28, http:/global .ihs.com/news/

S/A, "Green light for end-of-life-vehicles directive", Global Engineering Documents, vol.3, issu2, http://global .ihs.com/ news/a4-2.html

MPT,“Aprovechando materiales”, http://www.terra.com.mx/Automovil/articulo/ $101649 /$

http://noticias.vanguardia.com.mx, 25 de febrero de 2004

www.inare.org.mx/diferencia.htm 